\title{
Network coding based payload concatenation for relay-assisted V2V communications
}

\author{
Le Tien Trien ${ }^{a)}$ Koichi Adachi, and Yasushi Yamao \\ Advanced Wireless \& Communication Research Center (AWCC), The University of \\ Electro-Communications, Chofugaoka, Chofu-shi, Tokyo 182-8585, Japan
}

a) trien@awcc.uec.ac.jp

\begin{abstract}
A reliable vehicle-to-vehicle (V2V) communication is essential for safe and highly automated driving system. The use of roadside relay stations (RSs) has been studied to assist the V2V communication and improve its reliability. However, its improvement is limited by possible packet congestion at RSs. In order to mitigate the packet congestion at RSs, in this letter, we propose a network coding based payload concatenation forwarding with a payload sorting and selection algorithm. Large-scale computer simulations confirm that the proposed scheme remarkably improves the reliability of $\mathrm{V} 2 \mathrm{~V}$ communications.
\end{abstract}

Keywords: V2V, relay-assist, network coding, payload concatenation Classification: Wireless Communication Technologies

\section{References}

[1] H. Cheng and Y. Yamao, "Reliable inter-vehicle broadcast communication with sectorized roadside relay station," Proc. of IEEE VTC2013-Spring, Dresden, Germany, Jun. 2013. DOI:10.1109/VTCSpring.2013.6692735

[2] L. T. Trien, K. Adachi, and Y. Yamao, "Efficient CSMA/CA packet relayassisted scheme with payload combining for ITS V2V communication," J. Inf. Process., vol. 26, no. 1, pp. 11-19, Jan. 2018. DOI:10.2197/ipsjjip.26.11

[3] L. T. Trien and Y. Yamao, "Packet combining relay scheme with sectorized relay station for reliable ITS V2V communication," Proc. of IEEE VTC2015-Spring, Glasgow, UK, May. 2015. DOI:10.1109/VTCSpring.2015.7145993

[4] M. Li, Z. Yang, and W. Lou, "CodeOn: Cooperative popular content distribution for vehicular networks using symbol level network coding," IEEE Trans. J. Sel. Areas Commun., vol. 29, no. 1, pp. 223-235, Jan. 2011. DOI:10.1109/JSAC. 2011.110121

[5] M. H. Firooz and S. Roy, "Collaborative downloading in VANET using network coding,” Proc. IEEE ICC, Ottawa, Canada, Nov. 2012. DOI:10.1109/ICC.2012. 6364257

[6] Z. Wang, M. Hassan, and T. Moors, "Efficient loss recovery using network coding in vehicular safety communication," Proc. IEEE WCNC, Sydney, Australia, Jul. 2010. DOI:10.1109/WCNC.2010.5506247

[7] Y. Gao, G. G. M. N. Ali, P. H. J. Chong, and Y. L. Guan, "Network coding based BSM broadcasting at road intersection in V2V communication," Proc. IEEE VTC2016-Fall, Montreal, Canada, Sep. 2016. DOI:10.1109/VTCFall.2016. 
7881105

[8] "Standard for information technology—specific requirements - part 11: Wireless LAN medium access control (MAC) and physical layer (PHY) specifications," IEEE Std. no. 802.11-2016, Dec. 2016. DOI:10.1109/IEEESTD.2016.7786995

[9] M. Sepulcre, J. Gozalvez, and B. Coll-Perales, "Why 6 Mbps is not (always) the optimum data rate for beaconing in vehicular networks," IEEE Trans. Mobile Comput., vol. 16, no. 12, pp. 3568-3579, Dec. 2017. DOI:10.1109/TMC.2017. 2696533

\section{Introduction}

The shadowing and hidden terminal (HT) problems greatly limit the reliability of vehicle-to-vehicle communication (V2VC) around intersections. To alleviate those effects, a relay-assisted $\mathrm{V} 2 \mathrm{VC}$ scheme with sectorized receiving relay station (RS), namely SR-V2VC, has been proposed [1]. The packet reception rate at RS is improved by the sectorized receiving scheme, and hence the number of the packets to be relayed increases. This leads to a large number of awaiting packets in the transmit queue of RS. Thus, SR-V2VC scheme faces another issue when traffic load is high, i.e., packet drop due to a packet overflow/congestion at RS. If packet drop happens, the achievable gain obtained by SR-V2VC scheme may be limited. To alleviate this packet drop, a packet payload concatenating forwarding (PCF) has been proposed [2]. It has been shown that SR-V2VC with PCF (SR-V2VC/PCF) can remarkably reduce the packet drop probability and hence improve the packet delivery rate (PDR) [3]. However, as traffic further increases, packet congestion may still happen at RS.

Network coding (NC) is a promising solution to deal with the network congestion. As one application of NC in vehicular communications, a cooperative content downloading scheme using vehicle-to-infrastructure (V2I) communications has been proposed $[4,5]$. A few works have exploited the benefit of NC to safety related beaconing using V2VC [6, 7]. In [6], a packet loss recovery scheme using $\mathrm{NC}$ is proposed, and it is shown to be effective in a highway scenario. For the intersection environments, an $\mathrm{NC}$ based scheme that uses the intersection vehicle to encode and re-broadcast all its received beacon messages is proposed [7]. It is shown that total retransmission time as well as the number of rebroadcast slots can be reduced by the scheme. However, it is not guaranteed that PDR can be considerably improved, especially in multiple node environment. In this letter, an NC based PCF (PCF-NC) scheme with a payload sorting and selection algorithm is proposed to adapt to multiple node environment in an intersection. The proposed PCF-NC for SR-V2VC scheme (SR-V2VC/PCF-NC) can mitigate the congestion and further enhance the performance of $\mathrm{V} 2 \mathrm{VC}$.

\section{SR-V2VC/PCF-NC}

\subsection{Operational principle}

We consider the IEEE $802.11 \mathrm{p}$ based V2V broadcast communication [8]. In the normal PCF scheme, RS stores the received V2V payloads in a single queue and 


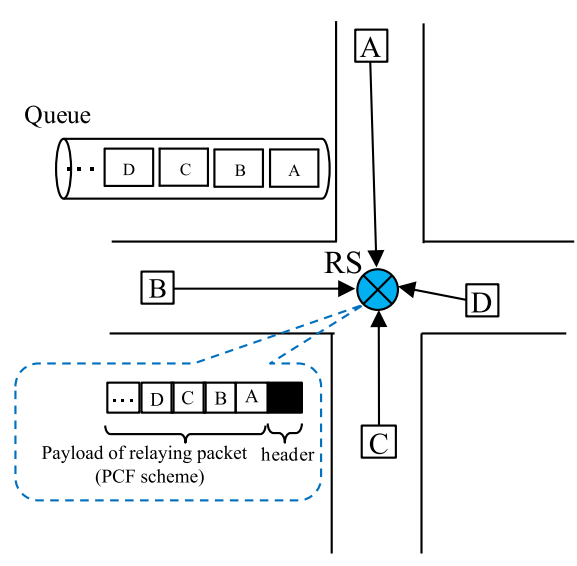

(a) PCF scheme

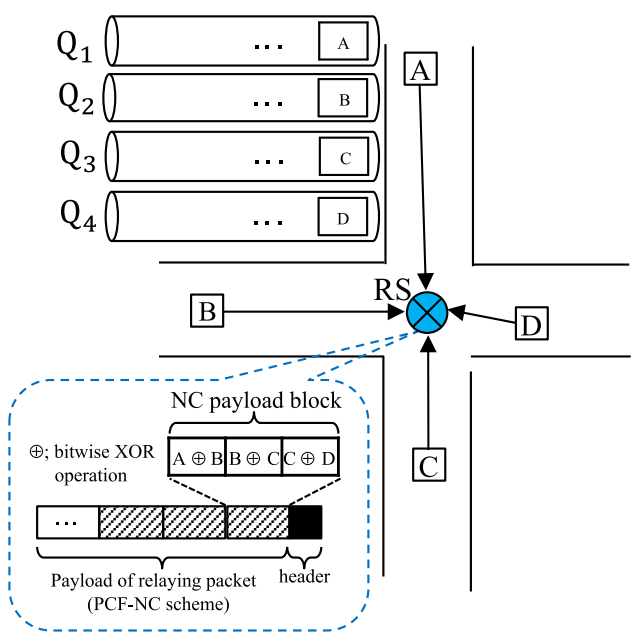

(b) PCF-NC scheme

Fig. 1. The operations of normal PCF scheme and PCF-NC scheme using a street-based grouping method with $G=4$.

concatenates payloads to generate a relaying packet as shown in Fig. 1(a). The payload concatenation process is initiated once either the number of payloads $k$ in the queue reaches the predetermined number $K$ or the waiting time of one of the payloads reaches the predetermined time $T_{\max }$ [2].

In the proposed PCF-NC scheme, all vehicle stations (VSs) are divided into $G$ groups and payloads of VSs from each group are stored in the corresponding queue. For example, if a street-based grouping method is adopted, $G$ can be set to the number of streets at the intersection as shown in Fig. 1(b). To generate a relaying packet, RS selects $G$ payloads from the queues and creates $(G-1)$ distinct payload pairs. Each pair will be encoded by XOR operation into a network coded payload. Let us denote the set of $(G-1)$ network coded payloads by NC payload block and define $J_{\min }=\min _{1 \leq g \leq G}\left(Q_{g}\right)$ with $Q_{g}$ being the size of queue $g$. Then $J_{\min } \mathrm{NC}$ payload blocks will be created from $\left(G \times J_{\min }\right)$ original payloads. The number of concatenated payloads $k_{\mathrm{NC}}$ for PCF-NC scheme results in

$$
k_{\mathrm{NC}}=(G-1) \times J_{\min }+\sum_{g=1}^{G}\left(\mathrm{Q}_{g}-J_{\min }\right) .
$$

Similar to the PCF scheme, a relaying packet will be generated if $k_{\mathrm{NC}}$ reaches $K$ or the waiting time reaches $T_{\max }$. The payload consists of $(G-1) \times J_{\min }$ network coded payloads and $\left(k_{\mathrm{NC}}-(G-1) \times J_{\min }\right)$ normal payloads. We will discuss the payload selection algorithm in detail in Sect. 2.2. Note that in the scenario of low traffic condition or extremely unbalanced vehicle distribution among groups, $J_{\min }$ may become 0 . Then the proposed PCF-NC scheme falls back to the conventional PCF scheme.

The header of the relaying packet needs to contain the values of $J_{\min }$ and $G$ so that receiving VSs (R-VSs) can determine the number of NC payload blocks in each relaying packet. R-VS first retrieves the values in the header of relaying packet and then decodes $J_{\min }$ NC payload blocks. All of $G$ original payloads in each NC payload block can be successfully decoded if at least one of them has been already received by R-VS via the direct communication with the transmitting VS (T-VS). 


\subsection{Payload sorting and selection algorithm}

Let $p_{g}(1 \leq g \leq G)$ denote the PDR of the original payloads via direct link to an $\mathrm{R}-\mathrm{VS}$. If none of the original packet payloads within the NC payload block has been successfully received, NC decoding failure happens. Thus, the decoding failure rate (DFR) of the NC payload block at R-VS is defined as

$$
\mathrm{DFR}=\prod_{g=1}^{G}\left(1-p_{g}\right) .
$$

Considering the street-based grouping method, one possible situation that faces decoding failure is when an NC payload block is solely composed of the payloads transmitted from T-VSs that are far from the R-VS. In such a situation, every $p_{g}$ $(1 \leq g \leq G)$ is low and hence the DFR becomes high. In order to avoid such a situation, we propose a sorting algorithm of payloads in each queue at RS. The sorting is performed based on the distance between RS and the T-VS of each payload, which can be obtained by decoding location information in payloads. The sorting order is alternated at certain interval to avoid unbalanced situation. As a result, the probability that all of T-VSs are far from specific R-VS decreases.

\section{Numerical results}

\subsection{Node layout and simulation set up}

In order to evaluate performance of the proposed PCF-NC scheme, computer simulations using Scenargie network simulator were conducted. We consider an urban environment with multiple intersections as shown in Fig. 2. One RS equipped with four-sectorized receiving antenna is installed at the center of each intersection. The distance between neighboring RSs is set to $300 \mathrm{~m}$. A total of 1176 VSs are uniformly distributed on the roads with four lanes. The radio transmission parameters follow [3]. The modulation/data rate of 16QAM/12 Mbps is employed [9]. The size of each packet payload is 100 bytes and its generation interval is $100 \mathrm{~ms}$. For the proposed PCF-NC scheme, all VSs are divided into 4 groups based on the streets they are located $(G=4)$. The maximum number of payloads $K$ in one relaying packet is set to 14 [2]. Thus, the proposed PCF-NC scheme can forward up to $18 \mathrm{~V} 2 \mathrm{~V}$ payloads in a single transmission opportunity, which is $28 \%$ higher than the conventional PCF scheme.

Direct V2V communication (D-V2VC), SR-V2VC/PCF, and SR-V2VC/PCF$\mathrm{NC}$ are compared in terms of broadcast packet delivery rate (BPDR), which is calculated for R-VSs in the evaluation area that spans $\pm 250 \mathrm{~m}$ along the roads from the T-VS of interest highlighted in blue-color in Fig. 2.

\subsection{Average DFR}

Fig. 3(a) shows the average DFR of the NC payload blocks transmitted from RS5 when R-VS is on the horizontal street. When R-VS is located near RS5, the PDRs via direct link at R-VS are relatively high. Thus, the average DFR is kept low. However, without sorting algorithm at RS, decoding failure happens when all the T-VSs that sent the original payloads are far from R-VS. Hence, the DFR slightly increases. With the sorting algorithm, the decoding failure decreases and the average DFR keeps lower than $1 \%$ for an area of R-VS of $\pm 225 \mathrm{~m}$ range. 


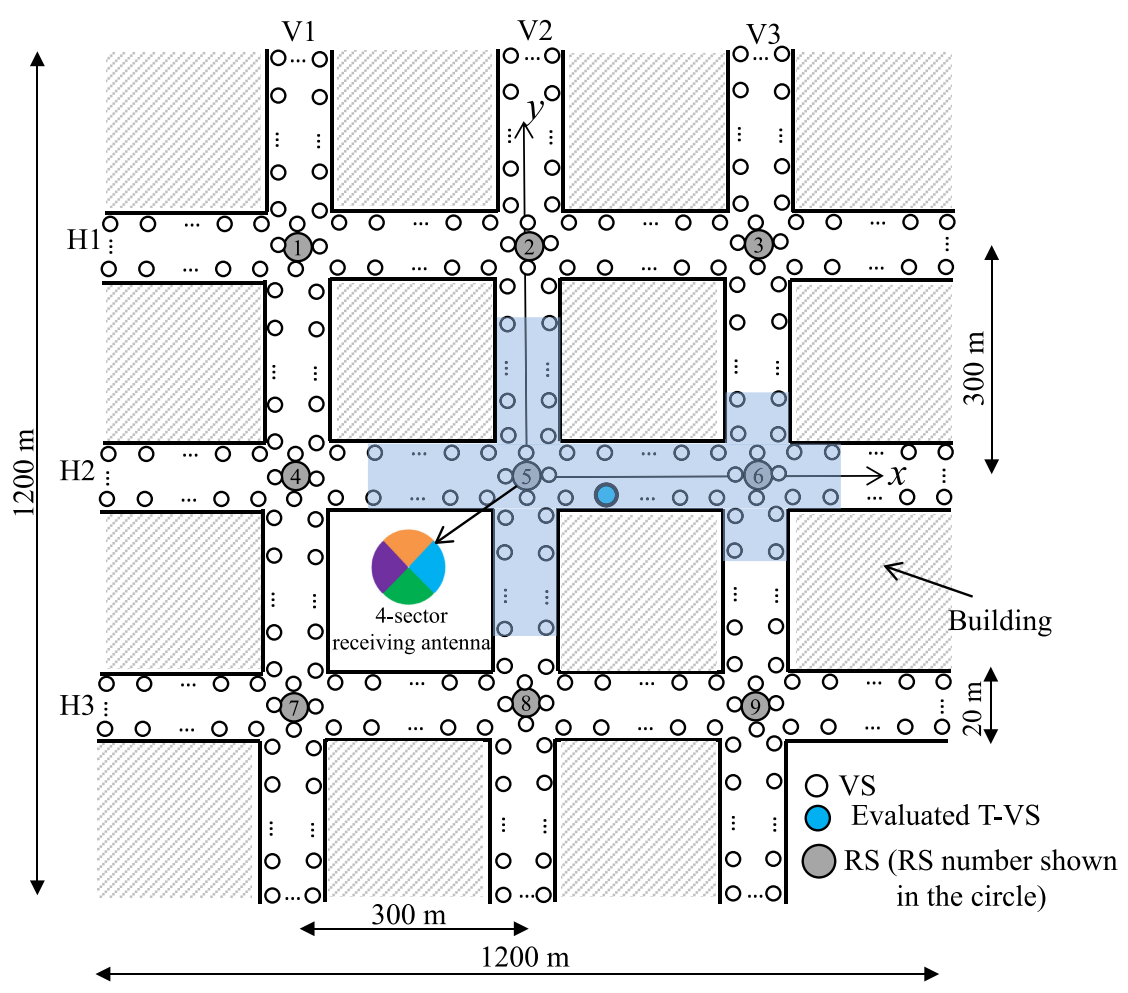

Fig. 2. Node layout model

When R-VS is located far from RS5, the probability that R-VS can successfully receive at least one of the payloads from T-VSs on different streets is quite low due to the large propagation loss. Thus, the average DFR mainly depends on the PDR of the original payload transmitted from T-VS on the same street as R-VS. As a result, the DFRs increase as R-VS moves far from RS5. However, the average DFR for the sorting case is lower than $4 \%$ for all locations of R-VS.

\subsection{BPDR performance}

Fig. 3(b) shows the BPDRs of the three V2V schemes. When T-VS is located near RS5, R-VSs in the evaluation area have LOS path to T-VS, and thus the BPDRs are high for all schemes. When T-VS moves away from RS5, BPDR of D-V2VC scheme rapidly decreases down to $63 \%$ at the intermediate point, i.e., $150 \mathrm{~m}$ from RS5. This is because the transmission from T-VS to R-VSs under NLOS conditions severely suffers from HT problem as well as shadowing. The SR-V2VC/PCF can improve BPDR up to $83 \%$ at the intermediate point by mitigating the effect of HT and compensating shadowing loss. We obtained from the simulations that the relay transmission rate at RS5, which is the ratio of the number of relay packets that are actually transmitted to the number of relay packets that are created by RS5, is only $76 \%$. This indicates that $24 \%$ of relay packets cannot be transmitted due to the queue overflow. On the other hand, the proposed SR-V2VC/PCF-NC alleviates the congestion issue and avoid packet drops, and hence improves the relay transmission rate up to $98 \%$. As a result, BPDR of SR-V2VC/PCF-NC scheme is higher than $90 \%$ for all locations of T-VS. This shows the effectiveness of the proposed scheme in mitigating the congestion issue. 


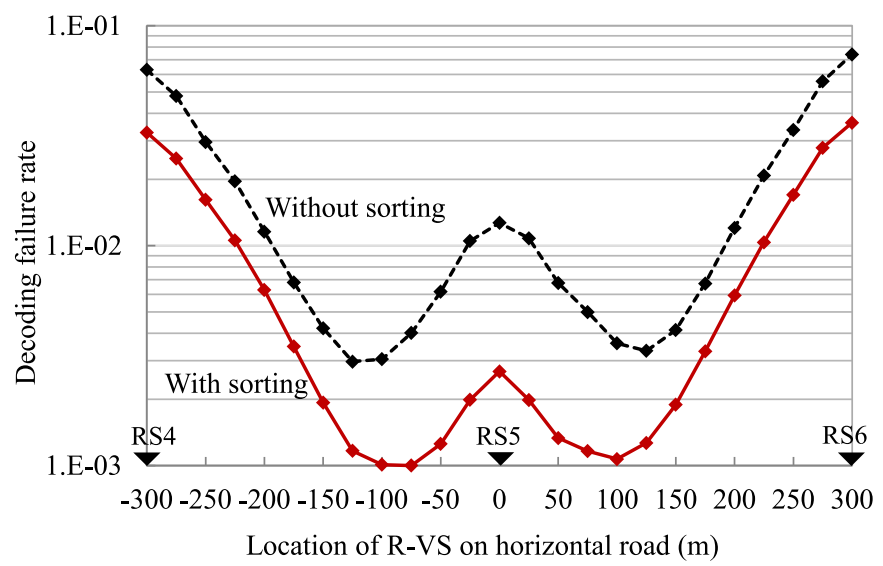

(a) Average DFR

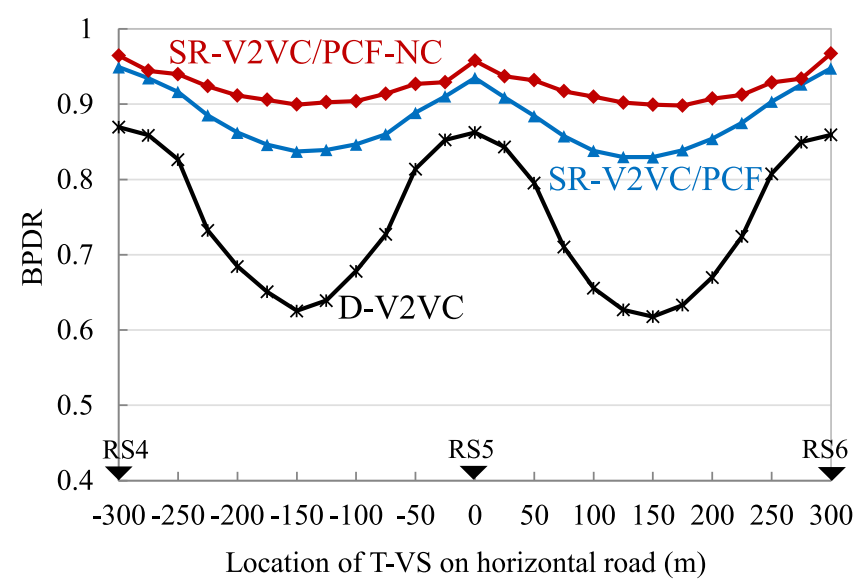

(b) BPDR

Fig. 3. Effectiveness of the proposed SR-V2VC/PCF-NC scheme

\section{Conclusion}

We have proposed a network coding based payload concatenation scheme to solve the congestion issue at relay stations for $\mathrm{V} 2 \mathrm{~V}$ communications. Large-scale simulation results showed that the proposed scheme can effectively mitigate the congestion issue, and remarkably improve the reliability of $\mathrm{V} 2 \mathrm{~V}$ communication. 